\title{
Multi-Objective Gain-Impedance Optimization of Yagi-Uda Antenna using NSBBO and NSPSO
}

\author{
Satvir Singh \\ SBS State Technical Campus \\ Ferozepur, Punjab [INDIA]
}

\author{
Etika Mittal \\ SBS State Technical Campus \\ Ferozepur, Punjab [INDIA]
}

\author{
Gagan Sachdeva \\ SBS State Technical Campus \\ Ferozepur, Punjab [INDIA]
}

\begin{abstract}
Biogeography-Based Optimization (BBO) is a population based algorithm which has shown impressive performance over other Evolutionary Algorithms (EAs). BBO algorithm is based on the study of distribution of biological organisms over space and time. YagiUda antenna design is most widely used antenna at VHF and UHF frequencies due to high gain, directivity and ease of construction. However, designing a Yagi-Uda antenna, that involves determination of optimal wire-lengths and their spacings, is highly complex and non-linear engineering problem. It further complicates as multiple objectives, viz. gain, and impedance, etc., are required to be optimized due to their conflicting nature, i.e., reactive antenna impedance increases significantly as antenna gain is intended to increase. In this paper Non-dominated Sorting BBO (NSBBO) is proposed and where standard and blended variants of $\mathrm{BBO}$ are investigated in optimizing six-element Yagi-Uda antenna designs for multiple objectives, viz., gain and impedance, where ranking of potential solutions is done using non-dominated sorting. The simulation results of BBO variants and Particle Swarm Optimization (PSO) are presented in the ending sections of the paper that depict clearly that NSBBO with blended migration operator is best option among all.
\end{abstract}

\section{Keywords:}

Non-dominated Sorting, Bio-geography Based Optimization (BBO), Particle Swarm Optimization (PSO), Yagi-Uda Antenna, Multi-Objective Optimization, Antenna Gain, Antenna Impedance

\section{INTRODUCTION}

Antenna is an electrical device which acts as an interface between free space radiations and transmitter (or receiver). The choice of an antenna depends on many factors such as gain, impedance, bandwidth, frequency of operation and Side Lobe Level (SLL), etc. Yagi-Uda antenna is amongst most popular antenna designs at VHF and UHF, i.e., $3 \mathrm{MHz}$ to $3 \mathrm{GHz}$ frequency range, due to its constructional ease and high gain, typically greater than $10 \mathrm{~dB}$. It is a parasitic linear array of parallel dipoles, one of which is energized directly by transmission line while the others act as a parasitic radiators whose currents are induced by mutual coupling. Therefore, characteristics of YagiUda antenna are affected by all of the geometric parameters of array.

A Yagi-Uda antenna was invented in 1926 by H. Yagi and S. Uda at Tohoku University in Japan [32, 36. Since its invention, continuous efforts have been put in optimizing its design for desired gain, impedance, SLL and bandwidth, etc., requirements using different optimization techniques based on traditional mathemat- ical approaches [24, 4, 8, 25, 7, 6, 9] and Artificial Intelligence (AI) techniques [16, 35, 34, 3, 19, 31, 30].

In 1949, Fishenden and Wiblin [15] proposed an approximate design of the antenna for maximum gain. In 1959, Ehrenspeck and Poehler have presented a manual approach to maximize the antenna by varying various lengths and spacings of its elements [14].

Later with the availability of computers and software at affordable prices, made it possible to optimize antennas numerically. Bojsen et al. in [4] proposed another optimization technique to calculate the maximum gain of Yagi-Uda antenna arrays with equal and unequal spacings between adjoining elements. Cheng et al. in [7] and [6] have used optimum wire lengths and their spacings to maximize the gain of the Yagi-Uda antenna. In [9], Cheng has proposed optimum design of Yagi-Uda antenna where antenna gain function is highly non-linear. In 1975, John Holland introduced Genetic Algorithms (GAs) as a stochastic, swarm based AI technique, inspired from natural evolution of species, to evolve optimal design of an arbitrary system based on certain cost function. Then many researchers investigated GAs to optimize Yagi-Uda antenna designs for gain, impedance and bandwidth as single objective problem [1, 16, 10] and multi-objective problem [35], 33, 18] without use of non-dominated sorting [12] in ranking different swarm solutions. Baskar et al. in [3], have optimized Yagi-Uda antenna using Comprehensive Learning Particle Swarm Optimization (CLPSO) and presented better results than other optimization approaches. Li has used Differential Evolution (DE) to optimize geometrical parameters of the antenna and illustrated the capabilities of the proposed method with several Yagi-Uda antenna designs in [19]. In [31], Singh et al. have explored another useful, stochastic global search and optimization technique named as Simulated Annealing (SA) to evolve optimal antenna design. In 2008, Dan Simon introduced yet another swarm based stochastic optimization algorithm based on the science of biogeography where features sharing among various habitats, i.e., potential solutions, is accomplished with migration operator and exploration of new features is done with mutation operator [28]. Singh et al. have presented BBO as a better optimization technique for Yagi-Uda antenna designs, in [30].

In this paper, use of BBO, Blended BBO and NSPSO algorithm along with non-dominated sorting are proposed and investigated to attain multiple objectives, i.e., (1) maximum gain and (2) only resistive impedance of $75 \Omega$, during Yagi-Uda antenna design optimization.

After this brief historical background survey, remaining paper is outlined as follows: Section 2 is dedicated to BBO algorithms. Section 3 explains PSO algorithm. In Section 4, Yagi-Uda an- 
tenna design parameters are discussed. Section 5 explains multiobjective problem formulation and non-dominated sorting algorithm. In Section 6, simulation results are presented and analyzed. Finally, conclusions and future scope have been discussed in Section 7.

\section{BIO-GEOGRAPHY BASED OPTIMIZATION}

BBO is a population based global optimization technique inspired from the science of biogeography, i.e., study of the distribution of animals and plants among different habitats over time and space. BBO results presented by researchers are better than other EAs [16, 33, 3, 23]. Originally, biogeography was studied by Alfred Wallace [2] and Charles Darwin [11] mainly as descriptive study. However, in 1967, the work carried out by MacAurthur and Wilson [20] changed this view point and proposed mathematical models for biogeography and made it feasible to predict number of species in a habitat, their migration, speciation, and extinction etc. over space and time. Habitats that are well suited residences for biological species are referred to have high Habitat Suitability Index (HSI) analogues to fitness in other EAs whose value depends upon many factors such as rainfall, diversity of vegetation, diversity of topographic features, land area, and temperature, etc. These features that characterize habitability are termed as Suitability Index Variables(SIVs). Each habitat, in a population of size $N P$, is represented by $M$-dimensional vector as $H=\left[S I V_{1}, S I V_{2}, \ldots, S I V_{M}\right]$ where $M$ is the number of SIVs to be evolved for optimal fitness given as $H S I=f(H)$.

The habitats with high HSI tend to have a large population of its resident species, that is responsible for more probability of emigration and less probability of immigration due to natural random behavior of species. Immigration is the arrival of new species into a habitat, while emigration is the act of leaving one's native region. On the other hand, habitats with low HSI tend to have low emigration probability, due to sparse population, however, they will have high immigration probability. Suitability of habitats with low HSI is likely to increase with influx of species from other habitats having high HSI. However, if HSI does not increase and remains low, species in that habitat go extinct that leads to additional immigration. For sake of simplicity, it is safe to assume a linear relationship between HSI and immigration and emigration probability and same maximum emigration and immigration probability, i.e., $E=I$, as depicted graphically in Figure 1.

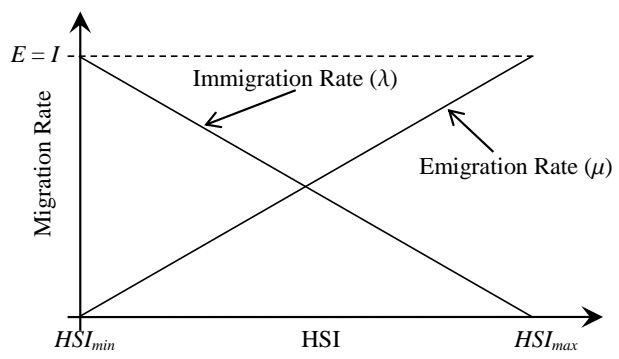

Fig. 1. Migration Curves

For $k$-th habitat values of emigration probability, $\mu_{k}$, and immigration probability, $\lambda_{k}$ are given by (1) and (2).

$$
\begin{gathered}
\mu_{k}=E \cdot \frac{H S I_{k}}{H S I_{\max }-H S I_{\min }} \\
\lambda_{k}=I \cdot\left(1-\frac{H S I_{k}}{H S I_{\max }-H S I_{\min }}\right)
\end{gathered}
$$

The migration of new species from high HSI to low HSI habitats may raise HSI of poor habitats as good solutions are more resistant to change than poor solutions whereas poor solutions are more dynamic and accept a lot of new features from good solutions. Following subsections describes the standard BBO [28] and blended BBO [29].

\subsection{Standard BBO}

2.1.1 Migration. Migration is a probabilistic operator that improves HSI of poor habitats by sharing features from good habitats. During migration, $i$-th habitat, $H_{i}$ (where $i=$ $1,2, \ldots, N P$ ) use its immigration rate, $\lambda_{i}$ given by (2), to probabilistically decide whether to immigrate or not. In case immigration is selected, then the emigrating habitat, $H_{j}$, is found probabilistically based on emigration rate, $\mu_{j}$ given by (1). The process of migration is completed by copying values of SIVs from $H_{j}$ to $H_{i}$ at random chosen sites, i.e., $H_{i}(S I V) \leftarrow H_{j}(S I V)$. The pseudo code of migration operator is depicted in Algorithm 1 .

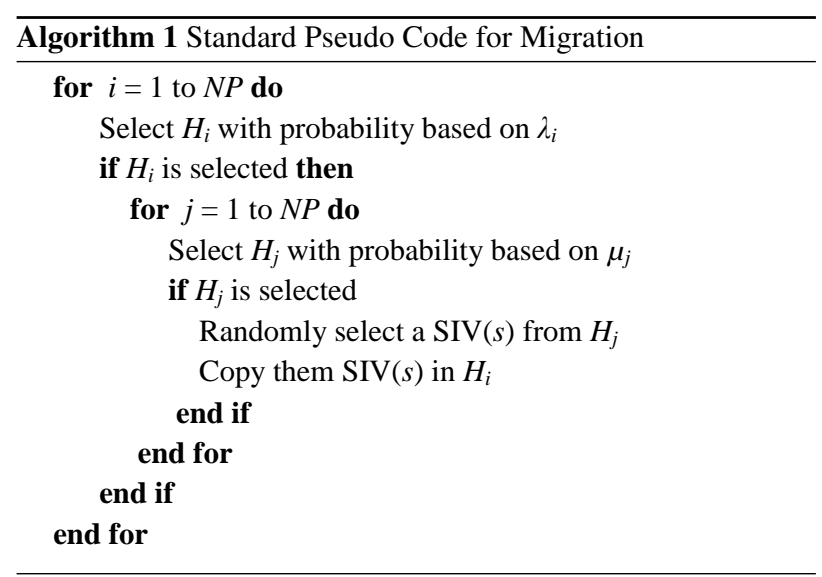

2.1.2 Mutation. Mutation is another probabilistic operator that modifies the values of some randomly selected SIVs of some habitats that are intended for exploration of search space for better solutions by increasing the biological diversity in the population. Here, higher mutation rates are investigated on habitats those are, probabilistically, participating less in migration process. The mutation rate, mRate, for $k$-th habitat is calculated as (3)

$$
\text { mate }_{k}=C \times \min \left(\mu_{k}, \lambda_{k}\right)
$$

where $\mu_{k}$ and $\lambda_{k}$ are emigration and immigration rates, respectively, given by (1) and (2) corresponding to $H S I_{k}$. Here $C$ is a scaling constant and equal to 3 . The pseudo code of mutation operator is depicted in Algorithm 2.

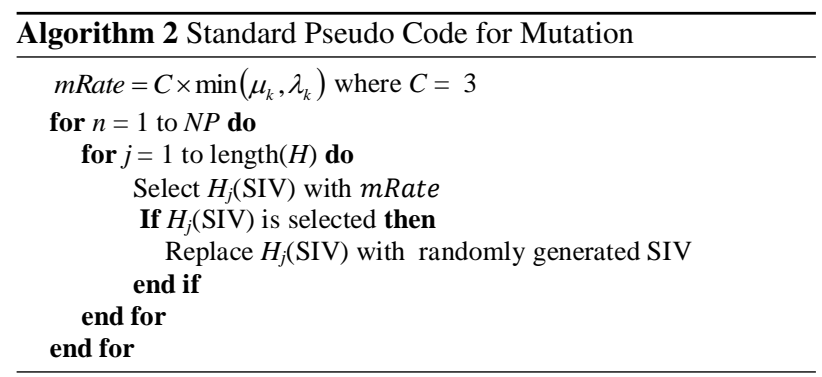




\subsection{Blended BBO}

Blended migration operator is a generalization of the standard BBO migration operator and inspired by blended crossover in GAs [21]. In blended migration, a solution feature of solution ImHbt is not simply replaced by a feature from solution $\mathrm{EmHbt}$ as happened in standard BBO migration operator. Instead, a new solution feature, $\operatorname{ImHbt}(\operatorname{SIV})$, solution is comprised of two components, i.e., $\operatorname{ImHbt}(S I V) \leftarrow \alpha \cdot \operatorname{ImHbt}(S I V)+(1-\alpha)$. $\operatorname{EmHbt}(S I V)$. Where $\alpha$ is a random number between 0 and 1 . The pseudo code of blended migration is depicted in Algorithm 3

Algorithm 3 Standard Pseudo Code for Blended Migration

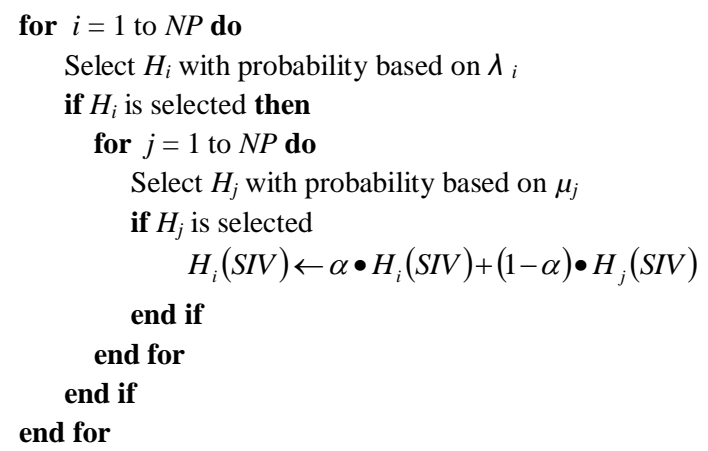

\section{PARTICLE SWARM OPTIMIZATION}

PSO algorithm is one of the stochastic swarm intelligence based global search algorithms. The motivation behind PSO algorithm is social behavior of animals, e.g., flocking of birds and fish schooling. PSO has its origin in simulations created to visualize the synchronized choreography of a bird flock by incorporating certain features like nearest-neighbor velocity matching and acceleration by distance [22, 27, 17, 13]. Later on, it was realized that the simulation could be used as an optimizer and resulted in the first simple version of PSO. In PSO, the particles have (1) adaptable velocities that determines their movement in the search space, (2) memory which enable them for remembering the best position in the search space ever visited. The position corresponding to the best fitness is known as past best, pbest, and the overall best out of all $N P$ the particles in the population is called global best, gbest. Consider that the search space is $M$-dimensional and $i$ th particle location in the swarm can be represented by $X_{i}=$ $\left[x_{i 1}, x_{i 2}, \ldots x_{i d} \ldots, x_{i M}\right]$ and its velocity can be represented by another $M$-dimensional vector $V_{i}=\left[v_{i 1}, v_{i 2}, \ldots . v_{i d} . ., v_{i M}\right]$. Let the best previously visited location position of this particle be denoted by $P_{i}=\left[p_{i 1}, p_{i 2}, \ldots . p_{i d} . ., p_{i M}\right]$, whereas, $g$-th particle, i.e., $P_{g}=\left[p_{g 1}, p_{g 2}, \ldots . p_{g d} . ., p_{g M}\right]$, is globally best particle location. Figure 2 depicts the vector movement of particle element from location $x_{i d}^{n}$ to $x_{i d}^{n+1}$ in $(n+1)$-th iteration that is being governed by past best location, $p_{i d}^{n}$, global best location, $p_{g d}^{n}$, and current velocity $v_{i d}^{n}$. Alternatively, the whole swarm is updated according to the equations (2) and (3) suggested by Shi \& Eberhart [26].

$$
v_{i d}^{m+1}=\chi\left(w v_{i d}^{m}+\psi_{1} r_{1}\left(p_{i d}^{m}-x_{i d}^{m}\right)+\psi_{2} r_{2}\left(p_{g d}^{m}-x_{i d}^{m}\right)\right)
$$

$$
x_{i d}^{m+1}=x_{i d}^{m}+v_{i d}^{m+1}
$$

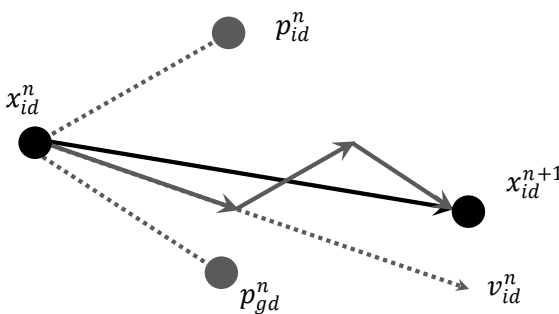

Fig. 2. Movement of $i$-th particle in 2-dimensional search space

Here, $w$ is inertia weight, $\psi_{1}$ is cognitive learning parameter, $\psi_{2}$ is social learning parameter and constriction factor, $\chi$, are strategy parameters of PSO algorithm, while $r_{1}$ and $r_{2}$ are random numbers uniformly distributed in the range $[0,1]$. Generally the inertia weight, $w$, is not kept fixed and is varied as the algorithm progresses. The particle movements is restricted with maximum velocity, $\pm V_{\max }$, to avoid jump over the optimal location as per search space requirements.

\section{ANTENNA DESIGN PARAMETERS}

Usually, Yagi-Uda antenna consists of three types of elements: (a) Reflector-biggest among all and is responsible for blocking radiations in one direction. (b) Feeder-which is fed with the signal from transmission line to be transmitted and (c) Directors-these are usually more then one in number and responsible of unidirectional radiations. Designing a Yagi-Uda antenna involves determination of wire-lengths and wire-spacings in between to get maximum gain, desired impedance and minimum SLL at an arbitrary frequency of operation. An antenna with $N$ elements requires $2 N-1$ parameters, i.e., $N$ wire lengths and $N-1$ spacings, that are to be determined. These $2 N-1$ parameters, collectively, are represented as a string referred as a habitat in $\mathrm{BBO}$ given as (6).

$$
H=\left[L_{1}, L_{2}, \ldots, L_{N}, S_{1}, S_{2}, \ldots, S_{N-1}\right]
$$

where $L_{s}$ are the lengths and $S_{s}$ are the spacing of antenna elements. An incoming field sets up resonant currents on all the antenna elements which re-radiate signals. These re-radiated signals are then picked up by the feeder element, that leads to total current induced in the feeder equivalent to combination of the direct field input and the re-radiated contributions from the director and reflector elements. This makes highly non-linear and complex relationships between antenna parameters and its characteristics like gain, impedance and SLL, etc.

Figure 3 depicts a typical six-wire Yagi-Uda antenna where all wires are placed parallel to $X$-axis and along $Y$-axis. Middle segment of the reflector element is placed at origin and excitation is applied to the middle segment of the feeder element.

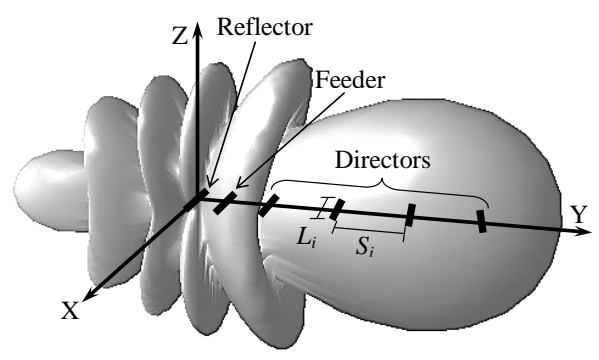

Fig. 3. Six-element Yagi-Uda Antenna 


\section{MULTI-OBJECTIVE OPTIMIZATION}

\subsection{Multi-objective Problems}

In single-objective optimization, optimal solution is easy to obtain as compared to multi-objective scenario where one solution may not exist which could be globally optimal with respect to all objectives. Objectives under consideration may be of conflicting in nature, i.e., improvement in one objective may cause declination in other objective(s). There exists a set of solutions which are the best tradeoff solutions important for decision making and are often superior to rest of solutions when all objectives are considered, however, inferior for one or more objectives. These solutions are termed as pareto-optimal solutions or non-dominated solutions and others are dominated solutions.

Multi-objective optimization problems result in pareto-optimal solutions instead of a single optimal solution in every run. Every solution from non-dominated set is acceptable as none of them is better than its counterpart. However, final selection of a solution is done by the designer based on nature of problem under consideration.

\subsection{Non-Dominated Sorting}

Problem, presented in this paper, of optimizing a six wire YagiUda antenna design has two objectives viz, (a) $75+j 0 \Omega$ antenna impedance and (b) maximum possible antenna gain. Subobjectives of antenna impedance, viz. real part, $R e$, and imaginary part, $I m$, are combined together to form single fitness function, as (7), that is required to be minimized.

$$
f_{1}=|R e-75|+|\operatorname{Im}|
$$

Whereas, second objective of gain maximization is also converted into minimization fitness function, $f_{2}$, given as (8)

$$
f_{2}=\frac{1}{\text { Gain }}
$$

Suppose every solution, in a swarm of $N P$ solutions, yields $f_{1_{k}}$ and $f_{2_{k}}$ as fitness values (where $k=1,2, \ldots, N P$ ), using (7) and (8), that belongs to a set of either non-dominated solutions, $P$, or dominated solutions, $D$. An $i$-th solution in set $P$ dominates the $j$-th solution in set $D$ if its satisfy the condition of dominance, i.e., $f_{1_{i}} \leq f_{1_{j}}$ and $f_{2_{i}} \leq f_{2_{j}}$, where both objectives are to be minimized. This condition of dominance is checked for every solution in the universal set of $N P$ solutions to assign it either $P$ set or $D$ set. Solution members of set $P$ form the first non-dominated front, i.e., the pareto optimal front, and then remaining solutions, those belong to set $D$, are made to face same condition of dominance among themselves to determine next non-dominated front. This process continues till all solutions are classified into different non-dominated fronts, as shown in Fig. 4. Preference order of solutions is to be based on designer's choice, however, here in this paper euclidian distance is determined from origin for every member solution in a non-dominated front and are picked up in ascending order. The pseudo code of Non-dominated sorting approach is depicted in Algorithm 4.

\section{SIMULATION RESULTS AND DISCUSSIONS}

As BBO and PSO are swarm based stochastic optimization algorithms, to present fair analysis, a six-wire Yagi-Uda antenna design is optimized for 10 times using 300 iterations and 30 habitats (particles). The universe of discourses to search optimal values of wire-lengths and wire-spacings are $0.40 \lambda-0.50 \lambda$ and $0.10 \lambda-0.45 \lambda$, respectively. However, cross-sectional radius and segment size for all wires are kept constant, i.e., $0.003397 \lambda$ and $0.1 \lambda$, respectively, where $\lambda$ is the wavelength corresponding to frequency of operation, i.e., $300 \mathrm{MHz}$. The $\mathrm{C}++$ programming
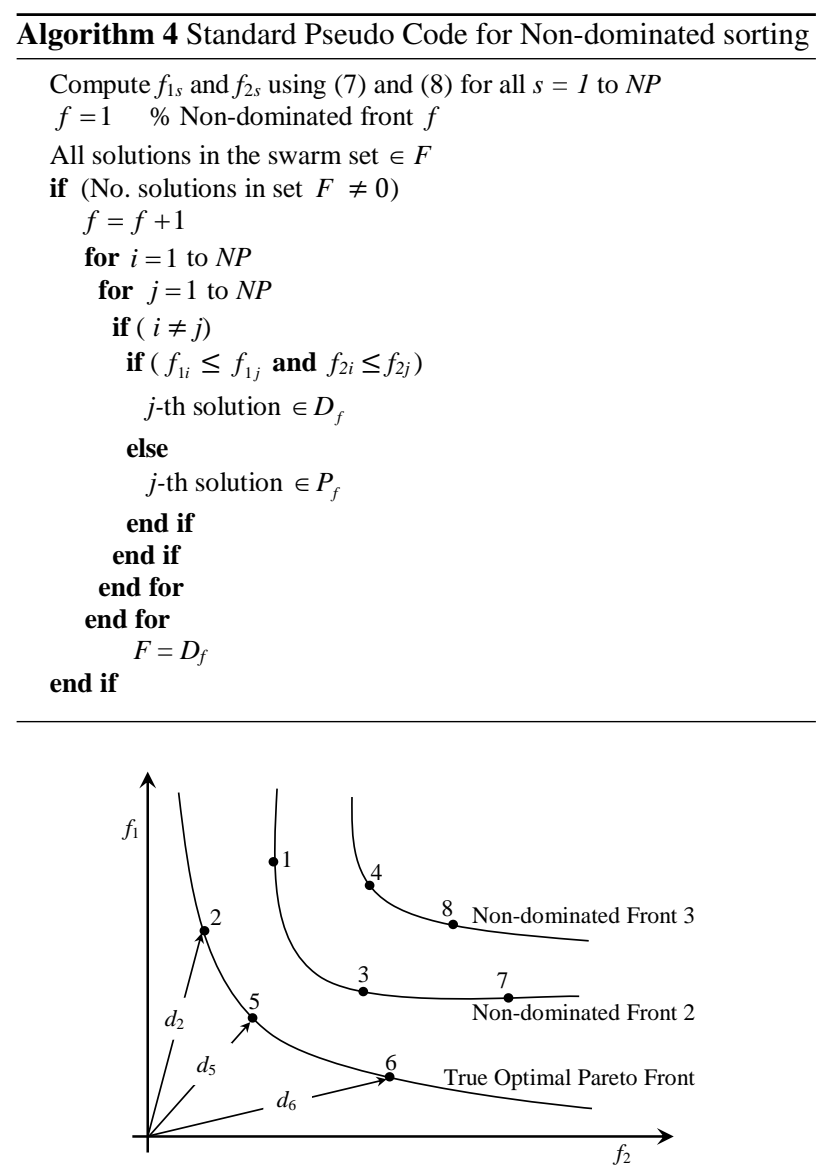

Fig. 4. Non-dominated sorting and pareto-fronts

platform is used for algorithm coding, whereas, method of moments based software, Numerical Electromagnetic Code (NEC2) [5], is called using system command to evaluate antenna designs. Both objectives, gain and impedance, are optimized using two fitness functions, given by (7) and (8).

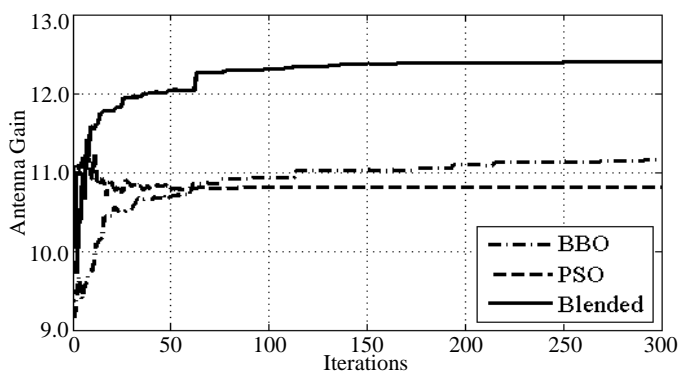

Fig. 5. Antenna Gain Convergence

Average of 10 Monte-Carlo simulation runs are plotted here to analyze convergence flow while achieving (i) maximum antenna gain, in Fig. 5, (ii) $R e=75 \Omega$, i.e., resistive antenna impedance of $75 \Omega$, in Fig. 6, and (iii) $I m=0 \Omega$, i.e., zero reactive antenna impedance, in Fig. 7. From the plots it can be observed that best compromised solution, during initial iterations, sometimes lead to poor solutions in terms of gain or impedance. However with increasing iteration number best compromised solution improves in aggregate that may, improve further, if maximum iteration number is kept high. The best antenna designs obtained during process of optimization and average results of 10 monte-carlo 


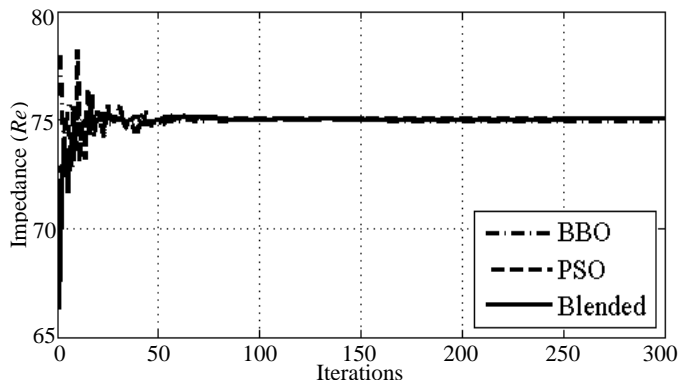

Fig. 6. Resistive Antenna Impedance

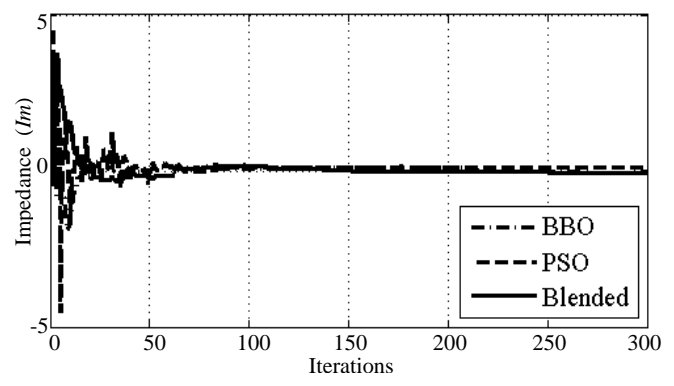

Fig. 7. Reactive Antenna Impedance

runs, depicted in Fig. 5 to 7 , after 300 iteration are tabulated in Table 1.

Table 1. The best antenna designs obtained during optimization

\begin{tabular}{|c|c|c|c|c|c|c|}
\hline \multirow[b]{2}{*}{ Element } & \multicolumn{2}{|c|}{ Standard BBO } & \multicolumn{2}{|c|}{ Blended BBO } & \multicolumn{2}{|c|}{ PSO } \\
\hline & Length & Spacing & Length & Spacing & Length & Spacing \\
\hline $1(\lambda)$ & 0.4732 & - & 0.4738 & - & 0.4525 & - \\
\hline $2(\lambda)$ & 0.4780 & 0.1979 & 0.4622 & 0.2185 & 0.4641 & 0.1917 \\
\hline $3(\lambda)$ & 0.4397 & 0.1631 & 0.4417 & 0.3929 & 0.4383 & 0.3546 \\
\hline $4(\lambda)$ & 0.4316 & 0.2735 & 0.4289 & 0.6546 & 0.4348 & 0.7201 \\
\hline $5(\lambda)$ & 0.4193 & 0.3902 & 0.4225 & 1.0289 & 0.4011 & 1.0408 \\
\hline $6(\lambda)$ & 0.4307 & 0.3360 & 0.4283 & 1.3835 & 0.4385 & 1.3909 \\
\hline Best Gain & \multicolumn{2}{|c|}{12.58} & \multicolumn{2}{|c|}{12.58} & \multicolumn{2}{|c|}{12.29} \\
\hline Best Imp. & \multicolumn{2}{|c|}{$74.9414+\mathrm{j} 0.0364 \Omega$} & \multirow{2}{*}{\multicolumn{2}{|c|}{ 75.2441-j0.0847 $\Omega$}} & \multicolumn{2}{|c|}{$68.1499-\mathrm{j} 2.9725 \Omega$} \\
\hline *Average Gain & \multicolumn{2}{|c|}{$11.16 \mathrm{dBi}$} & $12.40 \mathrm{dBi}$ & & \multicolumn{2}{|c|}{$10.81 \mathrm{dBi}$} \\
\hline *Average Imp. & \multicolumn{2}{|c|}{ 74.946-j0.024 $\Omega$} & \multicolumn{2}{|c|}{$75.050-\mathrm{j} 0.073 \Omega$} & \multicolumn{2}{|c|}{$75.048+\mathbf{j} 0.013 \Omega$} \\
\hline
\end{tabular}

\section{CONCLUSIONS AND FUTURE SCOPE}

In this paper, NSBBO and NSPSO algorithm are investigated for attaining multiple objectives, i.e., maximum gain and antenna impedance. It can be observed from simulation results that the NSBBO with blended migration presents better convergence flow in terms of achieving gain and only resistive impedance of $75 \Omega$ as compared to standard BBO and NSPSO over limited 300 iterations. Reasons for poor performance of PSO may include use of global best PSO model, where each particle learns from every other particle in the swarm and globally best particle, that is prone to get trapped in local optima. Investigation into other variants of $\mathrm{PSO}$ and $\mathrm{BBO}$ algorithms for improved performance is next on our agenda.

\section{REFERENCES}

[1] E.E. Altshuler and D.S. Linden. Wire-antenna Designs using Genetic Algorithms. Antennas and Propagation Magazine, IEEE, 39(2):33-43, 1997.
[2] A.Wallace. The Geographical Distribution of Animals. Boston, MA: Adamant Media Corporation, Two:232-237, 2005.

[3] S. Baskar, A. Alphones, P N Suganthan, and J J Liang. Design of Yagi-Uda Antennas using Comprehensive Learning Particle Swarm Optimisation. IEEE, 152(5):340-346, 2005.

[4] JH Bojsen, H. Schjaer-Jacobsen, E. Nilsson, and J. Bach Andersen. Maximum Gain of Yagi-Uda Arrays. Electronics Letters, 7(18):531-532, 1971.

[5] G. J. Burke and A. J. Poggio. Numerical Electromagnetics Code (NEC) method of moments. NOSC Tech. DocLawrence Livermore National Laboratory, Livermore, Calif, USA, 116:1-131, 1981.

[6] C. Chen and D. Cheng. Optimum Element Lengths for Yagi-Uda Arrays. IEEE Transactions on Antennas and Propagation, 23(1):8-15, 1975.

[7] D. Cheng and C. Chen. Optimum Element Spacings for Yagi-Uda Arrays. IEEE Transactions on Antennas and Propagation, 21(5):615-623, 1973.

[8] D. K. Cheng. Optimization Techniques for Antenna Arrays. Proceedings of the IEEE, 59(12):1664-1674, 1971.

[9] D. K. Cheng. Gain Optimization for Yagi-Uda Arrays. Antennas and Propagation Magazine, IEEE, 33(3):42-46, 1991.

[10] D. Correia, A. J. M. Soares, and M. A. B. Terada. Optimization of gain, impedance and bandwidth in Yagi-Uda Antennas using Genetic Algorithm. IEEE, 1:41-44, 1999.

[11] C. Darwin. The Orign of Species. New York : gramercy, Two:398-403, 1995.

[12] K. Deb, A. Pratap, S. Agarwal, and T. Meyarivan. A fast and elitist multiobjective genetic algorithm: Nsga-ii. Evolutionary Computation, IEEE Transactions on, 6(2):182197, 2002.

[13] R.C. Eberhart, Y. Shi, and J. Kennedy. Swarm Intelligence. Morgan Kaufmann Publisher, 2001.

[14] H. Ehrenspeck and H. Poehler. A New Method for Obtaining Maximum Gain from Yagi Antennas. IRE Transactions on Antennas and Propagation, 7(4):379-386, 1959.

[15] R. M. Fishenden and E. R. Wiblin. Design of Yagi Aerials. Proceedings of the IEE-Part III: Radio and Communication Engineering, 96(39):5, 1949.

[16] E. A. Jones and W. T. Joines. Design of Yagi-Uda Antennas using Genetic Algorithms. IEEE Transactions on Antennas and Propagation, 45(9):1386-1392, 1997.

[17] J. Kennedy and R. Eberhart. Particle swarm optimization. 4:1942-1948, 1995.

[18] Y. Kuwahara. Multiobjective Optimization Design of YagiUda Antenna. IEEE Transactions on Antennas and Propagation, 53(6):1984-1992, 2005.

[19] J. Y. Li. Optimizing Design of Antenna using Differential Evolution. IEEE, 1:1-4, 2007.

[20] R.H. MacArthur and E.O. Wilson. The Theory of Island Biogeography. Princeton Univ Pr, 1967.

[21] T. McTavish and D. Restrepo. Evolving Solutions: The Genetic Algorithm and Evolution Strategies for Finding Optimal Parameters. Applications of Computational Intelligence in Biology, 1:55-78, 2008.

[22] K.E. Parsopoulos and M.N. Vrahatis. Recent Approaches to Global Optimization Problems through Particle Swarm Optimization. Natural computing, 1(2):235-306, 2002.

[23] M. Rattan, M. S. Patterh, and B. S. Sohi. Optimization of Yagi-Uda Antenna using Simulated Annealing. Journal of Electromagnetic Waves and Applications, 22, 2(3):291299, 2008. 
[24] D. G. Reid. The Gain of an Idealized Yagi Array. Journal of the Institution of Electrical Engineers-Part IIIA: Radiolocation, , 93(3):564-566, 1946.

[25] L. C. Shen. Directivity and Bandwidth of Single-band and Double-band Yagi Arrays. IEEE Transactions on Antennas and Propagation, , 20(6):778-780, 1972.

[26] Y. Shi and R.C. Eberhart. Empirical Study of Particle Swarm Optimization. 3, 1999.

[27] Y. Shi et al. Particle Swarm Optimization: Developments, Applications and Resources. 1:81-86, 2001.

[28] D. Simon. Biogeography-based Optimization. IEEE Transactions on Evolutionary Computation,, 12(6):702-713, 2008.

[29] Satvir Singh and Gagan Sachdeva. Mutation Effects on BBO Evolution in Optimizing Yagi-Uda Antenna Design. In International Conference on Emerging Applications of Information Technology (EAIT2012), 2012.

[30] U. Singh, H. Kumar, and T. S. Kamal. Design of Yagi-Uda Antenna Using Biogeography Based Optimization. IEEE Transactions on Antennas and Propagation, , 58(10):33753379, 2010.
[31] U. Singh, M. Rattan, N. Singh, and M. S. Patterh. Design of a Yagi-Uda Antenna by Simulated Annealing for Gain, Impedance and FBR. IEEE, 1:974-979, 2007.

[32] Shintaro Uda and Yasuto Mushiake. Yagi-Uda Antenna. Maruzen Company, Ltd, 1954

[33] N. V. Venkatarayalu and T. Ray. Single and Multi-Objective Design of Yagi-Uda Antennas using Computational Intelligence. IEEE, 2:1237-1242, 2003.

[34] N.V. Venkatarayalu and T. Ray. Optimum Design of YagiUda Antennas Using Computational Intelligence. IEEE Transactions on Antennas and Propagation,, 52(7):18111818, 2004.

[35] H. J. Wang, K. F. Man, C. H. Chan, and K. M. Luk. Optimization of Yagi array by Hierarchical Genetic Algorithms. IEEE, 1:91-94, 2003.

[36] H. Yagi. Beam Transmission of Ultra Short Waves. Proceedings of the Institute of Radio Engineers, 16(6):715740,1928 